\section{AB0620 ORAL HEALTH IN PATIENTS WITH SYSTEMIC SCLEROSIS: AN EUSTAR CENTER EXPERIENCE}

C. Ancuta ${ }^{1,2}$, M. Antohe $^{2}$, E. Ancuta ${ }^{3}$, R. Chirieac ${ }^{4}$, C. lordache ${ }^{2}$.

${ }^{1}$ Rheumatology 2, Clinical Rehabilitation Hospital; ${ }^{2}$ University of Medicine and Pharmacy Grigore T Popa Iasi; ${ }^{3}$ Research Department, Elena Doamna Clinical Hospital; ${ }^{4}$ Sanocare Medical and Research Center, IASI, Romania

Background: Although the orofacial manifestations are commonly reported among patients with systemic sclerosis (SSc), only few studies have adequately addressed the issue of oral health-related quality of life (QoL) in such pathobiological settings.

Objectives: The aim was to characterize the oral health status (OHS) of adults with SSc and to compare with the general population.

Methods: A cross-sectional prospective observational study in 37 consecutive SSc in a EUSTAR cohort (EUSTAR 162 center) and 37 gender and age-matched controls without SSc.

A standardized oral exam meaning OHS (periodontal, dental, mucosal and microbial), oral health-related behaviors and oral HRQoL (Oral Health Impact Profile, OHIP) were evaluated in all recruited individuals, while oral manifestations such as size of oral aperture, oral dryness, manual dexterity for oral hygiene and $\mathrm{HRQ}$ oL only in SSc.

Multivariable regression analysis was done to evaluate association between SSc, oral abnormalities, oral health status and QoL.

Results: Overall, SSc had significantly reduced oral HRQoL compared with controls $(p<0.05)$.

We demonstrated lower resting salivary flow rates $(p<0.05)$ and $\mathrm{pH}(\mathrm{p}<0.05)$, reduced maximal mouth opening $(p<0.05)$, smaller interincisal distance $(p<0.05)$ in SSc group as compared to their controls. Moreover, despite comparable oral health-related behaviors (e.g. same level of daily tooth-brushing), the majority of SSc experienced caries $(p<0.05)$ and presented with more periodontal disease $(p<0.05)$ comprising periodontal pockets (pocket depth $P D>3 \mathrm{~mm})(p<0.05)$, clinical attachment level $(C A L) \geq 5.5 \mathrm{~mm}(p<0.05)$.

Finally, SSc was documented as a significant independent predictor of OHIP, missing teeth, periodontal disease, interincisal distances as shown by multivariate regression $(p<0.05)$.

Conclusions: SSc patients are at risk to develop impaired oral health and oral HRQoL compared with the general population.

Disclosure of Interest: None declared

DOI: 10.1136/annrheumdis-2017-eular.4115

\section{AB0621 DEVELOPMENT OF SYSTEMIC SCLEROSIS IN TRANSGENDERED FEMALES: A CASE SERIES}

C. Campochiaro, C. Fonseca, E. Derrett-Smith, V.H. Ong, C.P. Denton. Centre for Rheumatology and Connective Tissue Diseases, Royal Free Hospital and UCL Division of Medicine, London, United Kingdom

Background: Scleroderma (SSc) is an autoimmune connective tissue disease with a female preponderance (female to male ratio of 9.7:1) [1]. Sex hormones are thought to play a role in the susceptibility to autoimmune diseases [2].

Objectives: We report 3 cases of SSc in male-to-female transsexuals diagnosed following their male-to-female transition

Methods: Medical records of 3 patients diagnosed with SSc after male-to-female transition were reviewed. Disease features, hormonal therapies and surgical interventions related to gender reassignment were collected.

Results: At our tertiary University Hospital clinical service, 3 male-to-female transsexual patients were diagnosed with SSc following their surgery between May 1997 and October 2016. All 3 patients had started their transition before the onset of the disease and had not been diagnosed with any autoimmune disease before either starting the hormonal therapy required for the transition or before undergoing plastic surgery interventions. The first case was diagnosed with anti-RNA pol III +ve diffuse cutaneous SSc at the age of 35 yearsa year after the first surgical intervention for infected silicone buttock implants and approximately 5 years of hormonal therapy with combination of mestranol and norethisterone $\left(\right.$ Norinyl $\left.-1^{\circledR}\right)$. She experienced scleroderma renal crisis 2 years after the diagnosis and severe vascular involvement with frequent and severe digital ulcers (DU), Raynaud's phenomenon (RP), gastroesophageal reflux disease (GERD), pulmonary arterial hypertension and telangiectasiae. She required chronic dialysis and was treated with mycophenolate mofetil (MMF), rapamycin, bosentan and proton-pump inhibitors (PPI). She eventually died 8 years after the diagnosis. The second case was diagnosed at the of 49 years, approximately 6 months following her gender reassignmentand 5 years after having started hormonal therapy initially with conjugated estrogen isolated from pregnant mares $\left(\right.$ Premarin $\left.^{\circledR}\right)$ and later with ethinylestradiol and gestodene (Femoden ${ }^{\circledR}$ ). She developed ANA -ve, antiCCP antibody and rheumatoid factor positive limited cutaneous SSc/rheumatoid arthritis overlap syndrome and experienced severe DUs, RP, GERD, inflammatory arthritis and pulmonary fibrosis (PF). She was treated with MMF, PPI, calcium-channel blocker (CCB) and low-dose steroid therapy with good response. The third case was diagnosed at the age of 43 years, 2 years after having started triptorelin $\left(\right.$ Decapepty $\left.{ }^{\oplus}\right)$ and oestradiol valerate. She had not undergone any surgical intervention prior to her diagnosis. She developed anti-PM/Scl +ve limited cutaneous SSc/myositis overlap syndrome and experienced RP, GERD and PF. She has only been treated with $\mathrm{PPI}$ and $\mathrm{CCB}$ prior to her reconstructive surgery.
Conclusions: Although still debated, the role of sex hormones in triggering autoimmunity has been suggested in animal models and human studies [2]. The development of SSc in our case series supports the notion that altered profile of sex hormones may modulate autoimmunity in genetically susceptible individuals with distinct clinical and laboratory characteristics.

References:

[1] Barnes $\mathrm{J}$ et al. Epidemiology of systemic sclerosis: incidence, prevalence, survival, risk factors, malignancy, and environmental triggers. Curr Opin Rheumatol 2012.

[2] Pennell LM et al. Sex affects immunity. J autoimmun 2012.

Disclosure of Interest: None declared

DOI: 10.1136/annrheumdis-2017-eular.2912

\section{AB0622 RENAL RESISTIVE INDEX (RRI): PROPOSAL FOR AGE-ADJUSTED CUT-OFF VALUES IN SYSTEMIC SCLEROSIS PATIENTS}

C. Bruni ${ }^{1}$, V. Maestripieri ${ }^{2}$, G. Tesei ${ }^{1}$, M. Chiostri $^{3}$, C. Sambalino ${ }^{3}$,

S. Guiducci ${ }^{1}$, S. Bellando-Randone ${ }^{1}$, M. Boddi ${ }^{3}$, M. Matucci-Cerinic ${ }^{1}$.

${ }^{1}$ Department of Experimental and Clinical Medicine, Division of Rheumatology; ${ }^{2}$ Department of Internal Medicine, Division of Medicine for Care Complexity III, University of Florence; ${ }^{3}$ Department of Heart and Vessels, Division of Cardiology I, Azienda Ospedaliera Universitaria Careggi, Firenze, Italy

Background: Renal resistive index (RRI) by Doppler ultrasound, reflects changes in both renal vascular and tubular-interstitial compartments and systemic vascular compliance related to physiological (age) and pathological conditions among which hypertension, diabetes mellitus, hyperuricaemia, dyslipidaemia and chronic kidney disease play a major role [1]. Because of the age-related changes in RRI reported in literature $[2,3]$ the use of a 0.70 cut-off to detect renal damage, as proposed [4], was questioned: renal injury in younger decades (<60yrs) may occur also for RRI value $<0.70$ and be underestimated. In systemic sclerosis (SSc), RRI was previously correlated with disease duration, glomerular filtration rate and nailfold-videocapillaroscopy pattern [5-7], although tested on small samples and not investigating the possible confounding role of age-related RRI values.

Objectives: to describe RRI in a larger scleroderma population and to test both the fixed $0.70 \mathrm{RRI}$ cut-off and age-adjusted cut-offs in reflecting renal and other disease-related organ damage.

Methods: SSc patients attending classified according to ACR/EULAR 2013 criteria were enrolled. Data on renal arteries Doppler ultrasound (RRI), autoantibodies status and biochemical tests for renal function/damage, subset and extent of skin fibrosis, instrumental assessment for internal organ involvement were collected and analysed as appropriate with SPSS vers 20.0. Considering that age-adjusted mean values were higher in the SSc population compared to literature values for the general population, we created SSc-specific age-adjusted pathologic cut-offs dividing our SSc population in quartiles and considering RRI values above the 75th percentile as pathologic (Table 1).

Results: 190 SSc patients (age $56.3 \pm 15.0$ years, disease duration $6 \pm 8,20$ men) were eligible for the study. In the SSc population significant positive correlations between RRI and age, as well as significant associations between RRI and above mentioned general population comorbidities [1], were confirmed. When considering absolute value of RRI, the 0.70 pathologic cut-off and age-adjusted cut-offs validated in the general population [1], only renal function, systolic PAP, DLCO and late nailfold scleroderma pattern were associated with RRI (Figure 1). Pathologic RRI identified according to age cut-offs could not detect early renal damage, but was significantly associated with various fibrotic [interstitial lung disease $(p=0.015)$, tendon friction rubs $(p=0.032)$, skin fibrosis vs no skin involvement $(p<0.001)$,

\begin{tabular}{lc}
\hline Age & Proposed SSc age-adjusted pathologic cutoff \\
\hline 1st quartile, $\leq 48$ years & $\geq 0,68$ \\
2nd quartile, 49-58 years & $\geq 0,69$ \\
3rd quartile, 59-67 years & $\geq 0,75$ \\
4th quartile, $\geq 68$ years & $\geq 0,77$ \\
\hline
\end{tabular}

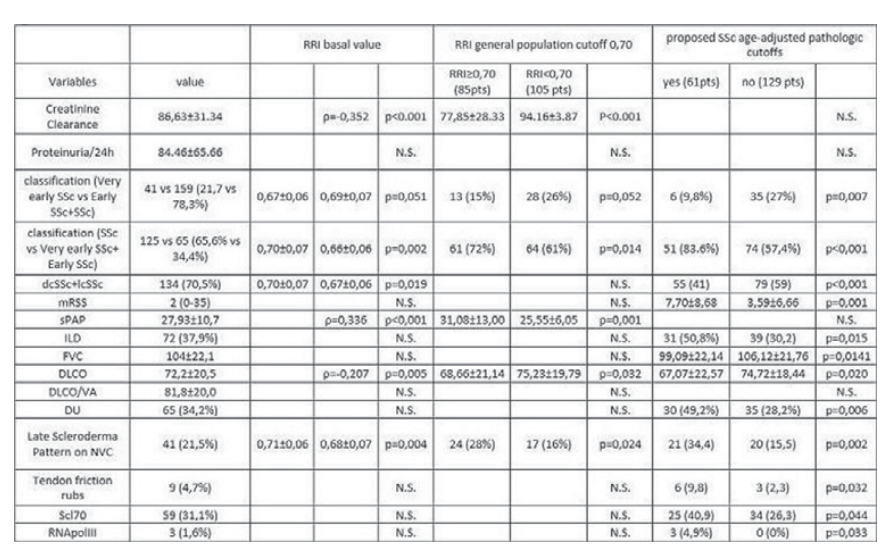

